\title{
Percepción del apoyo social en adultos mayores pertenecientes a las instituciones denominadas Centros de Vida"
}

\section{Perceptions of Social Support among Older Adults Living in the so-called Centros de Vida Institutions}

\author{
Shirley Fernández Aragón \\ ORCID: 0000-0003-4213-3736 \\ Corporación Universitaria Rafael Núñez, \\ Cartagena, Colombia \\ Diana Cáceres Rivera \\ ORCID: 0000-0002-4751-6173 \\ Universidad Cooperativa de Colombia \\ Yolima Manrique-Anaya* \\ ORCID: 0000-0002-3986-7870 \\ Corporación Universidad Rafael Núñez, \\ Cartagena, Colombia \\ Universidad de Cartagena, Colombia
}

Recibido: 22 enero de 2018 Revisado: 7 de junio de 2019 Aceptado: 2 de septiembre 2019

\section{Resumen}

En los adultos mayores las redes de apoyo social son determinantes para su estado de salud, su ausencia incrementa el riesgo de trastornos físicos y psicológicos. El objetivo de esta investigación fue describir la percepción del apoyo social en los adultos mayores pertenecientes a los denominados Centros de Vida en la ciudad de Cartagena (Colombia). Fue un estudio descriptivo-transversal en una muestra de 320 individuos de una población de 2.308 adultos mayores; la información se recolectó a través de la Escala Multidimensional Percepción de Apoyo Social. Socio-demográficamente la edad estuvo entre 80 - 89 años (32.8\%), masculinos (50.9\%), casados (29.7\%) y en unión libre (30.3\%), sin formación educativa (47.8\%), sin hijos $(90.6 \%)$ y nietos solo $(84.1 \%)$, hipertensos (48.8\%). La percepción de apoyo social más frecuente fue excelente $(34.4 \%)$, sobre todo con la familia $(45.3 \%)$ y allegados $(48.1 \%)$. Los adultos mayores perciben un excelente apoyo social en las instituciones.

Palabras claves: Adultos mayores; apoyo social; percepción; hogares geriátricos.

Artículo de investigación. Citar como: Fernández, A. S., Cáceres, R. D., \& Manrique-Anaya, Y. (2020). Percepción del apoyo social en adultos mayores pertenecientes a las instituciones denominadas Centros de Vida. Diversitas: Perspectivas en Psicología, 16(1), 55-64. Dol: https://doi. org/10.15332/22563067.5541

Correspondencia: Yolima Manrique Anaya, Corporación Universitaria Rafael Núñez, Cartagena, Colombia. Dirección postal: Cra 4 N $36-70$ sede principal. Correo electrónico: yolima.manrique@curnvirtual.edu.co 


\section{Abstract}

Networks of social support are determining factors for the health of older adults, since their absence increases the risk of physical and psychological disorders. This research aimed to describe the perception of social support among older adults living in the so-called Centros de Vida [Life Centers] in Cartagena, Colombia. It was a descriptive-transversal study, with a sample of 320 individuals from a population of 2,308 resident seniors. Information was collected through the Perception of Social Support Multidimensional Scale. Socio-demographically, participants were between 80 and 89 years of age $(32.8 \%)$, male $(50.9 \%)$, married $(29.7 \%)$, in free union $(30.3 \%)$, lacking in educational background $(47.8 \%)$, childless $(90.6 \%)$, having just grandchildren (84.1\%), and suffering from hypertension $(48.8 \%)$. The most frequent perception of social support was excellent (34.4\%), especially with family (45.3\%) and relatives (48.1\%). In conclusion, older adults perceive excellent social support in these institutions.

Keywords: older adults; social support; perception; life centers.

\section{Introducción}

En la etapa de vida del ser humano conocida como la adultez mayor se hacen visibles los cambios anatómicos, funcionales y psicológicos que acarrea el proceso de envejecimiento, el cual es irreversible en todo ser vivo. Así, se afirma que la vejez se comprende como un proceso del ciclo vital (infancia, pubertad, adolescencia, madurez), que se distingue por sus propios atributos, lo que hace que en algunos individuos sea percibida como más agradables que para otros, esto teniendo en cuenta las actitudes que se asumen (de modo individual, familiar, amigos, comunidad, entre otros) y las circunstancias de vida (Cuadra, Medina, \& Salazar, 2016).

A nivel internacional, y de acuerdo a la organización mundial de la salud (oms) en la actualidad se vive más tiempo en todo el mundo, en la medida en que por primera vez en la historia, la mayor parte de la población tiene una esperanza de vida igual o superior a los 60 años. Para 2050 se espera que la población mundial en esa franja de edad llegue a los 2000 millones, un aumento de 900 millones con respecto al año 2015. Actualmente existen $125 \mathrm{mi}-$ llones de personas con 80 años o más, mientras que para 2050 habrá un número casi igual de personas en este grupo de edad (120 millones) solamente en China, y 434 millones de personas en todo el mundo. De igual modo, un $80 \%$ de todas las personas ma- yores vivirá en países de ingresos bajos y medianos (OMS, 2018).

En Colombia según el Estudio Nacional de Salud, Bienestar y Envejecimiento (2015), la cifra de personas mayores de 60 años representó el $11 \%$ de la población colombiana. Es decir 5.2 millones de personas, siendo aquellos de 60-69 años el 57.2\%, los de 70-79 años el $30.2 \%$, y los de 80 y más el $12.6 \%$. Para el año 2020 se estima que el porcentaje de adultos mayores aumente al $12.5 \%$, que equivaldría a 6.5 millones de personas; y para el 2050 se proyecta en un $23 \%$, es decir, 14.1 millones de adultos mayores (Minsalud, 2015).

Al hacer referencia al contexto de la ciudad de Cartagena (Colombia), en 2015 había 107.257 adultos mayores, de los cuales 46.608 eran hombres y 60.649 eran mujeres (Alcaldía Mayor de Cartagena de Indias, 2012). Los mayores de 65 años son en la actualidad el $7.0 \%$ del total de la población, cifra superior al $1.0 \%$ que existía en 2005. La cantidad de adultos mayores por cada 100 individuos entre 15-64 años pasó de $8.51 \%$ en 2005 a $10.4 \%$ en 2014 . A partir de esto se muestra un envejecimiento progresivo de la población local (Dadis 2014).

Con lo anterior y dada la progresión de la población adulta mayor, se debe resaltar el aporte que hacen a la sociedad de diferentes maneras, ya sea en el 
seno de sus familias, en la comunidad local o en la sociedad en general. No obstante, las oportunidades que se tendrán al envejecer dependerán en gran medida de la salud, pues a edades mayores, se presenta disminución de la capacidad física y mental (Arias, 2013).

Un aspecto fundamental para que lo mencionado se lleve a cabo de forma satisfactoria es a través del apoyo social (Arias, 2013). Este hace referencia a la percepción a partir de su interrelación en redes, de sentir que es amado y cuidado, valorado y estimado (García, 2013). En la etapa adulta mayor las redes de apoyo social son uno de los aspectos más relevantes que afectan el estado de salud. Se ha demostrado que la falta de apoyo, sobre todo por parte de la familia, incrementa el riesgo en la aparición de enfermedades y que las escasas redes de apoyo social acentúan el riesgo de diversos trastornos psicológicos (Cunurana, 2009).

De este modo, el apoyo social podría proteger a las personas mayores de los efectos secundarios a eventos estresantes y además afectar positivamente la salud de las personas al suministrar recursos (ayuda económica, material, información), mejor acceso al cuidado de salud y regulación de los hábitos de vida. En consecuencia, el apoyo social ayuda al adulto mayor a mantener autonomía e independencia dentro de su contexto familiar y sociocultural, lo que es fundamental para su bienestar (Pereira \& Pavarini, 2012).

La evidencia empírica local, que ha abordado el apoyo social en el adulto mayor desde la perspectiva de la funcionalidad familiar, sugiere que existe buena percepción de elementos propios de la vida familiar. Los elementos favorables son la adaptabilidad, la cooperación, la satisfacción, la afectividad y la capacidad resolutiva por parte de los adultos mayores, que se constituye en un factor de importancia dado que se pueden utilizar como factores protectores para evitar alteraciones propias de esta etapa de la vida, tales como la depresión, la ansiedad, la desesperanza, la falta de participación, entre otros (Acuña, Barrios, Martínez, Taborda, \& Vargas, 2015). Estos elementos se podrían interpretar como percepción del apoyo social, sin embargo, no se presenta de forma diferenciada que grupo de sujetos proporcionan el apoyo (es decir, la familia, los amigos u otros significativos), ni tampoco se cuantifica el apoyo que cada uno de estos aporta.

Partiendo de estas consideraciones y tomando en cuenta la relevancia que tiene el apoyo social para los adultos mayores, además de la limitada información que existe para el ámbito local de la ciudad de Cartagena, en la presente investigación se describió la percepción de apoyo social en los adultos mayores pertenecientes a las instituciones denominadas centros de Vida de la ciudad de Cartagena, durante el primer y segundo semestre de 2017.

\section{Materiales y Métodos}

Fue un estudio de naturaleza cuantitativa descriptiva y de corte transversal.

\section{Participantes}

La población estuvo conformada por 2.308 adultos mayores residentes en los 30 Centros de Vida de la ciudad de Cartagena de Indias, con los que se estimó una muestra de 320 sujetos (error de $5 \%$ y un nivel de confianza de $95 \%$ ) elegido mediante un muestreo abierto por conveniencia según el numero de adultos mayores residentes en cada uno de los centros de vida.

Los criterios de inclusión fueron: (1) Adultos mayores que residan al interior de los Centros de Vida, (2) que no presentaran alteraciones mentales y (3) disposición a participar en el estudio.

\section{Instrumentos}

Se aplicó la Escala Multidimensional de Percepción de Apoyo Social (MSPSS) diseñada por Zimet, Dahlem, Zimet y Farley (1988), compuesta de 12 ítems que miden el apoyo social percibido. Esta escala consta de 3 subescalas: familia, otros significativos $y$ amigos. La escala de medida es de tipo Likert con 4 alternativas para cada uno de los ítems ( $1=$ Casi nunca; $2=$ A veces; $3=$ Con frecuencia, $4=$ Siempre). El instrumento presenta una confiabilidad total de $a=0.86$ (alfa de Cronbach). Cabe señalar, que un mayor puntaje, en cada una de las escalas antes se- 
ñaladas, indica la presencia de mayor Apoyo Social. Las preguntas se clasificaron así: familia (ítems 3, 4, 8,11 ), otros significativos (ítems $1,2,5,10$ ) y amigos (ítems 6, 7, 9, 12).

La recolección de la información se efectuó directamente y de manera presencial en cada centro de vida objeto de estudio, se tuvo en cuenta la disponibilidad de los participantes y el cumplimiento de los criterios de inclusión; seguidamente se le explicaba la finalidad del estudio y se daba a conocer el consentimiento informado; luego de su aceptación para participar, se procedió a la aplicación del instrumento; este proceso demoraba aproximadamente entre 45 a 60 minutos por participante, los cuales en su totalidad fueron 320 distribuidos en 30 centros de vida de la ciudad. Este procedimiento fue realizado por los investigadores y por personas previamente entrenadas en el tema y en el instrumento. Todo este proceso se dio hasta completar el tamaño de la muestra estimada.

Las consideraciones éticas se fundamentaron en la Resolución 8430 de 1993 (Minsalud, 1993), y los participantes firmaron el consentimiento informado.

\section{Resultados}

\section{Aspectos sociodemográficos de los adultos mayores}

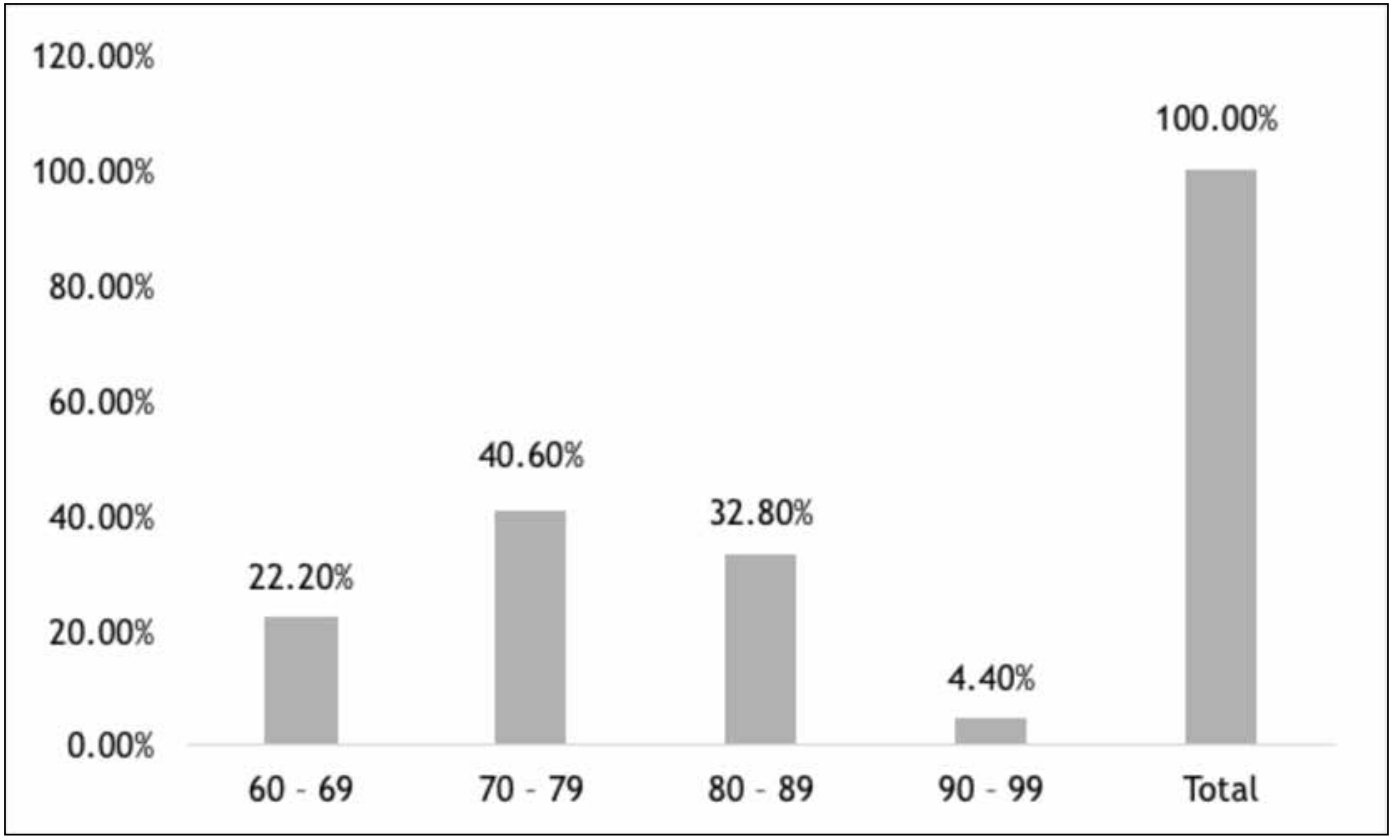

Figura 1. Rangos de edades de los participantes.

Fuente: elaboración propia. 
Con relación al género se apreciaron participaciones relativamente similares, siendo el masculinos del $50.9 \%$, y el femenino del $49.1 \%$. A partir de esto se puede afirmar claramente que se garantizó la representatividad tanto de hombres como de mujeres en el estudio.
Sobre el estado civil se puede observar el predominio del $30.3 \%$ del total de encuestados en unión libre es decir que mantienen una relación afectiva con otra persona sin estar casadas y en menor porcentaje con un $29.7 \%$ la condición de casados, lo cual evidentemente obedece a la edad de estas personas.

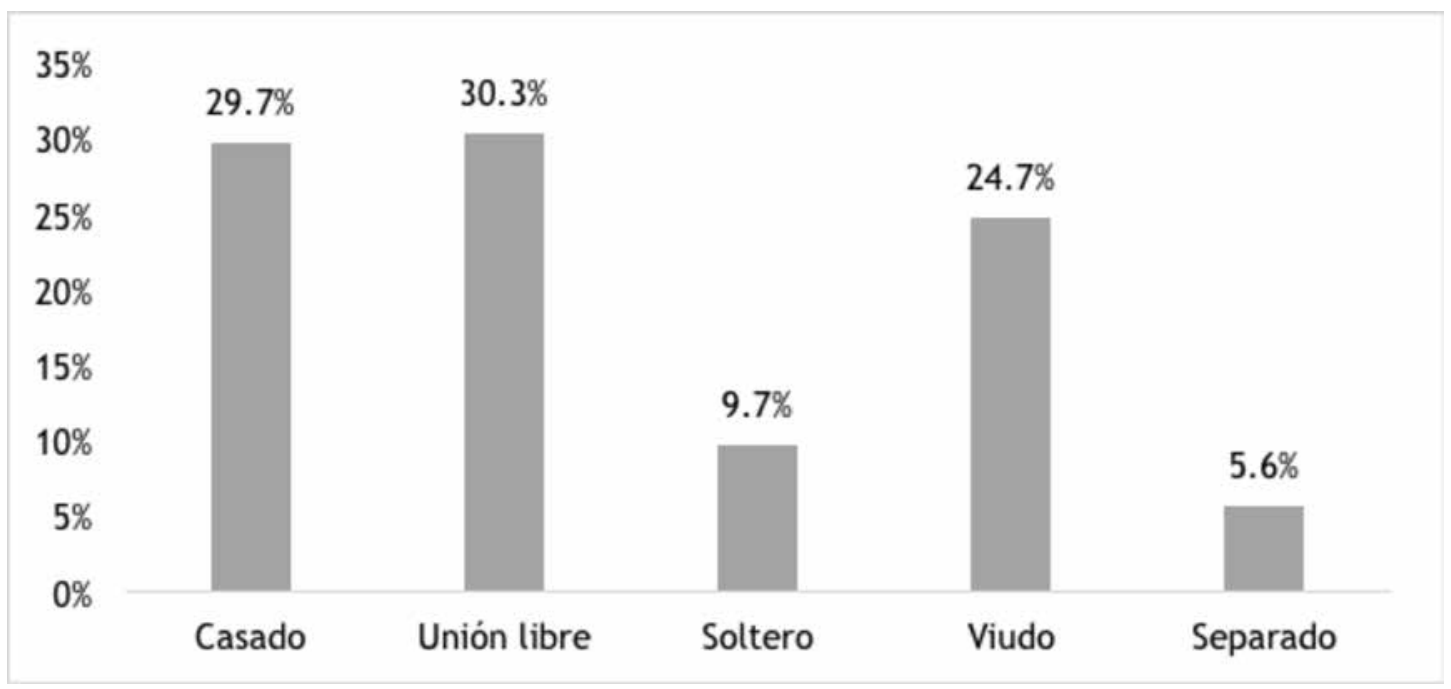

Figura 2. Distribución del estado civil

Fuente: elaboración propia.

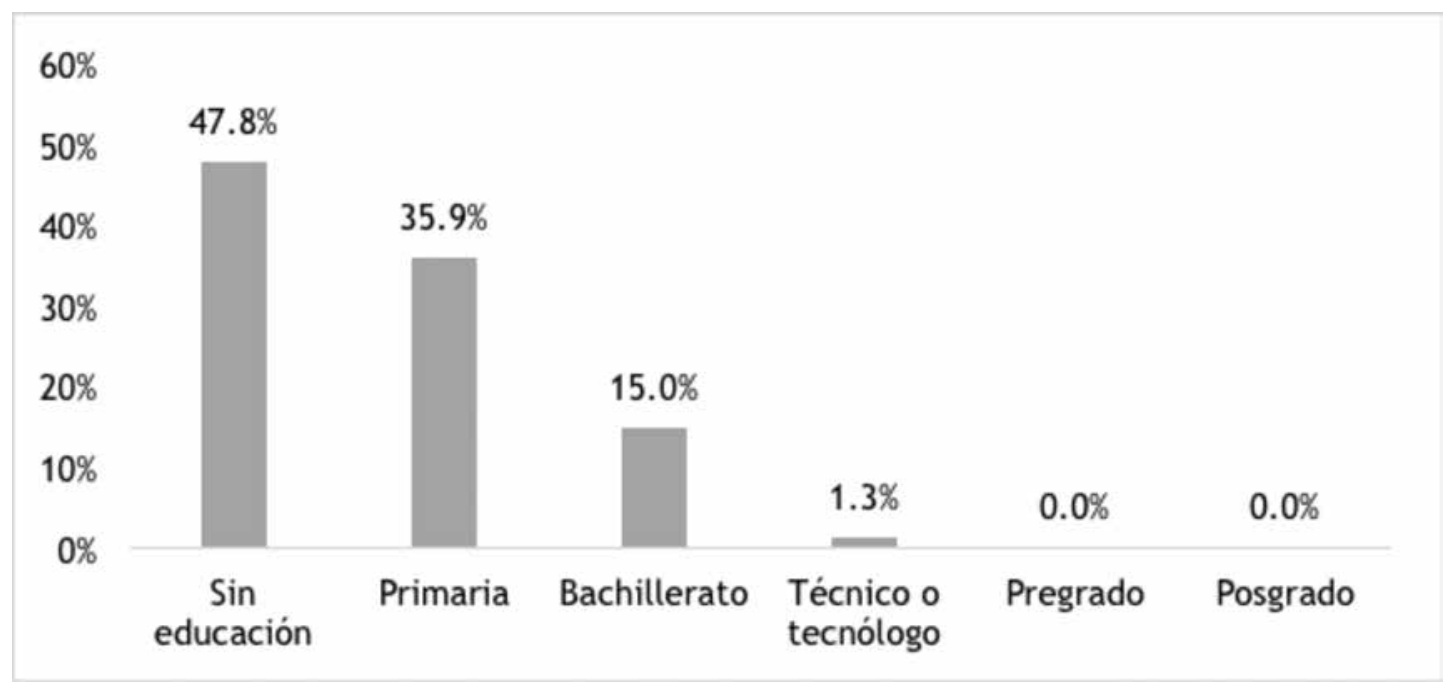

Figura 3. Distribución del nivel educativo

Fuente: elaboración propia. 
Este bajo nivel educativo se debe a que los sujetos que hicieron parte de este estudio corresponden a una generación temprana, cuyo nacimiento tuvo lugar antes de la segunda mitad del siglo xx, momento en el cual la educación no había alcanzado la valoración, difusión, ni obligatoriedad en que la actualidad posee.

En lo que corresponde a la ascendencia de los adultos mayores, puede indicarse que la gran mayoría reconoció tener hijos (90.6\%). Así mismo, un alto porcentaje mencionó tener nietos (84.1\%). Finalmente, la enfermedad con mayor prevalencia entre los sujetos, correspondió a la hipertensión arterial, la cual se presentó en el $48.8 \%$ del total; estuvo seguida de la diabetes (15.3\%), y de la artritis (3.8\%). En menor proporción existieron casos de osteoporosis $(1.3 \%)$ y asma (0.3\%).

\section{Apoyo social percibido por los adultos mayores}

Se logró establecer que la percepción de apoyo social por parte de los adultos mayores resultó ser predominantemente "excelente", con un $34.4 \%$ del total. También hubo algunos cuya percepción fue "buena" (31.6\%), seguidos de quienes lo consideraron "regular" (23.1\%) y, en menor proporción, los que tienen la idea de que el apoyo social es "malo" (10.9\%).

Dados estos resultados, se buscó conocer cuáles fueron los atributos que distinguían a los adultos mayores que perciben su apoyo social como excelente, bueno, regular, o malo.

\section{Resultado general según aspectos sociodemográficos de los adultos mayores}

La separación de la percepción sobre el apoyo social en términos de las características sociodemográficas de los adultos mayores, dejó entrever diversos elementos relevantes. Con relación a la variable edad, se detectó que en todos los grupos etarios prevaleció una percepción excelente del apoyo social, sobre todo entre las personas con 60 - 69 años (33.8\%) y 80 - 89 años (35.2\%), aunque también se destacaron las respuestas de buena percepción entre los de 90 - 89 años (32.4\%) y 70 - 79 años (21.5\%).

Cuando se separó a la población objetivo según su género, se encontró que las mujeres, en comparación con los hombres tuvieron una percepción de apoyo social excelente más frecuente (35.7\% frente a 33.1\% en hombres). También llamó la atención que, entre los adultos mayores hombres, fue mayor la mala percepción de apoyo social (14.7\%) respecto a las mujeres $(7 \%)$.

De acuerdo con los datos recolectados, también llamó la atención el hecho de que los adultos mayores que se reconocieron como solteros, tuvieran en mayor medida una percepción de apoyo social excelente (48.4\%). Lo cual contrastó con la situación de los separados, entre quienes tuvo lugar el menor porcentaje de percepción excelente (16.7\%). Además, en este grupo se presentó la mayor prevalencia de percepción regular (33.3\%) y mala (16.7\%).

Un aspecto a destacar con base en la separación por niveles educativos, consistió en que los adultos mayores con formación de técnicos o tecnólogos tuvieron la peor valoración del apoyo social, en la medida en que fue sobre todo malo $(50 \%)$ y regular (25\%). Finalmente se encontró que los sujetos con bachillerato tuvieron, sobre todo percepción de apoyo excelente (41.7\%) y escasamente malo (2.1\%), mientras que en los de ninguna formación educacional prevaleció la mala percepción (13.1\%), aunque presentaron con cierta frecuencia valoración excelente (34\%).

Tal como se esperaba, los adultos mayores que reconocieron tener hijos presentaron una percepción de apoyo social usualmente excelente (36.2\%) y buena (32.8\%), en comparación con los que no, ya que en estos se destacó la percepción regular (33.3\%) y mala (30\%). Algo similar tuvo lugar para los sujetos que indicaron tener nietos, pues generalmente su percepción de apoyo social se caracterizó por ser excelente (36.8\%) y buena (32.7\%), en contraste con los que no tienen nietos, pues su percepción de apoyo social recurrentemente es mala (17.6\%) y regular (35.3\%).

El último de los aspectos sociodemográficos tenido en cuenta correspondió al padecimiento de enfermedades. Al tener en cuenta las dos enfermedades 
más prevalentes, es decir la hipertensión arterial y la diabetes, se encontraron las siguientes particularidades. En primera instancia, la percepción de apoyo social resultó ser frecuentemente mejor entre quienes no padecen ninguna de las dos patologías crónicas, pues fue excelente y buena en el $35.4 \%$ y $34.8 \%$, respectivamente, de los que no presentan hipertensión, y en el $36.2 \%$ y el $32.8 \%$ de aquellos sin diabetes. A diferencia de lo anterior, los que sí sufren hipertensión catalogaron en una proporción menor el apoyo social excelente y bueno en $33.3 \%$ y $28.2 \%$, y los que presentan diabetes en $24.5 \%$ y $24.5 \%$, respectivamente.

En segundo lugar, cuando se tuvieron en cuenta las respuestas menos favorables sobre la percepción de apoyo social por parte de los adultos mayores, se halló igualmente que quienes no padecen diabetes en menor proporción lo catalogaron como malo $(10.3 \%)$ en contraste con los que sí la padecen (14.3\%). Así mismo, en el grupo de los no hipertensos la percepción de mal apoyo fue algo menor (9.8\%) cuando se comparó los que sí lo son (12.2\%).

\section{Resultado por ámbitos}

La última consideración para abordar el perfil de percepción del apoyo social entre los adultos mayores, consistió en tener en cuenta tres ámbitos o grupos de personas que les resultan allegadas. En primer lugar, se les indagó sobre la familia, al respecto, prevaleció la percepción de excelente apoyo ya que así lo reconoció el $45.3 \%$, así como el calificativo de bueno, con el $22.8 \%$. En este orden de ideas, estas personas son conscientes de que su familia trata de ayudarle, les otorga ayuda y apoyo emocional que requieren, sumado a esto, pueden conversar sus problemas con el grupo familiar, a la vez que les ayudan a tomar decisiones.

En segunda instancia se observaron las opiniones respecto a la manera en que consideran el apoyo social que proviene de los amigos. En este caso, la participación del calificativo malo fue la más destacada, en tanto que alcanzó el $32.5 \%$, sumado a que la valoración de regular, también alcanzó una cifra notable, con $24.7 \%$. Esto en cierta medida contrastó con el $25.6 \%$ que opinó que los amigos los apoyan de modo excelente.
En tercer y último lugar, sobre el grupo de otros allegados (que se refiere a personas que no son propiamente amigos, sino más que todo conocidos, familiares no tan cercanos, entre otros sujetos que de algún modo tiene vínculos con los adultos mayores), se encontró que el apoyo que proporcionan suele ser excelente (48.1\%), aunque también bueno (22.2\%), pero en menor medida, se valoró como regular $(18.1 \%)$ o malo $(11.6 \%)$.

\section{Discusión}

La realización de la presente investigación mostró la percepción del adulto mayor en relación con el apoyo social recibido en los Centros de Vida, siendo este evaluado excelente. Lo anterior está alineado con la premisa formulada por Arias (2013), respecto a que el apoyo social en sentido amplio, y en particular en los adultos mayores, ejerce un rol muy importante en tanto que contribuye a proveer sentido a la vida, aporta retroalimentación cotidiana acerca de las desviaciones de la salud, propicia una serie de actividades personales que se asocian positivamente con sobrevida, y que se orientan al cuidado y a la promoción de la salud.

En cuanto a los resultados que muestra esta investigación de la percepción del apoyo entre excelente y bueno, se detectaron similitudes con otros estudios como el de Figueroa, Soto y Santos (2016), en el cual estimaron que un $75 \%$ de su población objetivo presentaba una excelente percepción de apoyo social. Así mismo, Jiménez, Jara y Miranda (2012), indican que el $62.92 \%$ de los sujetos de su investigación percibieron buenos niveles de apoyo social.

Por otro lado, se documentó que los adultos mayores calificaron como excelente el apoyo social que reciben específicamente de su familia (45.3\%) y de otros allegados (48.1\%), mientras que consideraron como malo el que reciben de sus amigos (32.5\%). Esto fue consistente con la información que dieron a conocer Polizzi y Arias (2014) respecto a los vínculos que brindan mayor satisfacción en la red de apoyo social de los adultos mayores; en donde identificaron que los hijos fueron los familiares con la mayor participación (41.7\%) incluso otros familiares (18.3\%) y la pareja. Pero a diferencia del presente estudio, los autores mencionados posicionaron en segundo 
lugar a los amigos (23.3\%). De acuerdo con Merodio, Rivas y Martínez (2015), este resultado obedece a que la "familia cumple un papel importante en el apoyo instrumental de las personas mayores, el cónyuge y los hijos son los principales proveedores de ayuda económica y cuidados; y es el cónyuge con quien las personas mayores cuentan incondicionalmente, ya que con los hijos pueden hacerlo solo en un plazo breve o frente a situaciones específicas" (p.51). Dicha afirmación guarda relación con los datos aportados en esta investigación, pues se observó excelente percepción de apoyo entre los adultos mayores casados (35.8\%), en unión libre (33\%), y con hijos (36.2\%).

En relación con las otras dimensiones del instrumento como apoyo familiar y colaboradores (Jiménez, Baillet, Ávalos, \& Campos, 2016), también se han descrito como alto el apoyo recibido por parte de sus familiares, en similitud con Guzmán, Huenchuan y Montes de Oca (2003), quienes reconocen que en gran medida la familia es percibida como fuente esencial de apoyo. Así mismo, Merodio, Rivas y Martínez (2015) reconocen que la familia proporciona una cantidad de apoyo importante, en opinión de los adultos mayores, sin embargo, es más relevante aún aquel que procede de la pareja y de los hijos.

Otro dato relevante tuvo que ver con que el estado civil, específicamente los adultos mayores solteros refirieron una percepción de apoyo social principalmente de tipo excelente $(48.4 \%)$, y aunque en aquellos con pareja también se destacó esta valoración, fue claramente una proporción algo más baja, tanto entre los casados (35.8\%) como en unión libre (33\%). Esta situación resultó ser opuesta la reportada con los datos de Vivaldi y Barra (2012), en donde se puede apreciar que el apoyo social percibido por los adultos mayores suele ser mejor por parte de los que tienen pareja (promedio $=40.87$ ) en comparación con los que no tienen (promedio = 38.06). Cabe añadir que, para el grupo de los separados, la percepción de apoyo predominante fue tanto mala (16.7\%) como regular (33.3\%), ante esto, los autores citados indican que "las personas que se encuentran sin pareja estable (como los separados o divorciados) presentarían más dificultades para adaptarse física y psicológicamente, porque también presentan menor apoyo social percibido, el cual sería un importante recurso de afrontamiento del estrés psicosocial" (p.25)

En el análisis del género se detectó que ambos presentaron predominio de percepción excelente de apoyo social, en el caso de los masculinos esta proporción ascendió al 33.1\% y en los femeninos al 35.7\%. Al comparar este hallazgo con el de Merodio, Rivas y Martínez (2015), se observan semejanzas en la medida en que los hombres reciben buen apoyo (sobre todo de sus familias) (44.1\%), pero contrasta con el hecho de que el apoyo recibido por las mujeres es sobre todo bajo (62\%).

\section{Recomendaciones}

Es recomendable que a los familiares y a otros allegados se les concientice de la importancia sobre continuar proporcionando el apoyo social que actualmente les brindan a los adultos mayores. Es decir, es necesario mantener la percepción mayoritariamente excelente o buena que dieron los encuestados. De igual modo es necesario que los familiares, allegados y amigos de los adultos mayores que perciben el apoyo social como malo o regular, sean capacitados, instruidos y concientizados sobre sus deberes y responsabilidades para con estas personas, se espera que con ello puedan mejorar el apoyo que proporcionan.

\section{Conclusiones}

Con respecto al apoyo social se pudo determinar que, en general, los adultos mayores pertenecientes a los Centros de Vida de la ciudad de Cartagena, lo perciben frecuentemente como excelente y como bueno, en relación al que proporcionan sus familiares y otros allegados. Esto contrastó con la valoración del apoyo de los amigos, el cual suele ser entre malo y regular.

En términos sociodemográficos se pudo establecerse que los adultos mayores estuvieron representados por sujetos de diversas edades, lo que permitió lograr representatividad de la gran mayoría de esta población. De igual modo, hubo tanto sujetos masculinos como femeninos con participaciones similares. Entre tanto, estas personas generalmente 
tenían pareja, pues se declararon principalmente casados y en unión libre. Sin embargo, otros indicaron ser viudos, lo que era de esperar, dada la edad de la población estudiada. Con respecto al nivel educativo se concluyó que prevalecieron los adultos mayores sin formación educativa, así como los que cursaron primaria; esto se esperaba en una población que vivió su infancia y juventud en un momento en el que la educación no era masiva ni tampoco obligatoria en la sociedad. A su vez, se concluyó que el hecho de tener hijos y nietos fue lo común entre los encuestados. Para el caso de los padecimientos y enfermedades, los resultados llevaron a confirmar que la hipertensión, y en menor medida la diabetes, fueron las más recurrentes.

La población estudiada reconoció poseer una red adecuada de relaciones sociales, mediante las cuales reciben el soporte necesario, tanto en situaciones cotidianas, como de crisis en la vida. Esto se reflejaría en el hecho de que reciben seguridad, refuerzo, afirmación, validación y estímulo, bajo un ambiente de respeto positivo incondicional y cuidado, todo lo cual se vería reflejado en una salud física y mental satisfactoria.

Lo anterior se contrastó con las valoraciones de quienes no tienen a familiares cercanos, en los cuales prevalecieron las opiniones de apoyo malo y regular.

\section{Agradecimientos}

A las estudiantes Leidy Paola Castellar Agudelo, Roxana Camila de Voz Palomino y Tanay Guerrero Acosta.

\section{Referencias}

Acuña, I., Barrios, Y., Martínez, N., Taborda, E., \& Vargas, G. (2015). Percepción de la funcionalidad familiar en adultos mayores de los centros de vida de la ciudad de Cartagena. (Tesis de pregrado). Cartagena: Universidad de Cartagena.

Alcaldía Mayor de Cartagena de Indias. (2012). Política pública de envejecimiento y vejez 20122024. Cartagena: Gaceta Municipal.
Arias, C. (2013). El apoyo social en la vejez: La familia, los amigos y la comunidad. Revista Kairós Gerontología, 6(4), 313-329.

Cuadra, A., Medina, E., \& Salazar, K. (2016). Relación de bienestar psicológico, apoyo social, estado de salud física y mental con calidad de vida en adultos mayores de la ciudad de Arica. Revista Interdisciplinaria de Filosofía y Psicología, 11(35), 56-67.

Cunurana, R. (2009). Redes de apoyo social percibido por el adulto mayor del Centro de Salud San Francisco del distrito Gregorio Albarracín Tacna. (Tesis de pregrado). Tacna, Perú: Universidad Nacional Jorge Basadre Grohmann.

Departamento Administrativo Distrital de Salud, DADIS. (2014). Perfil epidemiologico del distrito de Cartagena. Cartagena de Indias: Departamento Administrativo Distrital de Salud (DADIS). Disponible en: http://www.dadiscartagena.gov.co/images/docs/saludpublica/per fil_epidemiologico_2014.pdf.

Figueroa, L., Soto, D., \& Santos, N. (2016). Calidad de vida y apoyo social percibido en adultos mayores. Revista de Ciencias Médicas de Pinar del Río, 20(1), 554-561.

García, F. (2013). Autopercepción de Salud y Envejecimiento. Ciencia e Innovación en Salud, 1(1), 69-77.

Guzmán, J., Huenchuan, S., \& Montes de Oca, V. (2003). Redes de apoyo social de personas mayores: Marco teórico conceptual. Simposio viejos y viejas (págs. 35-70). Santiago de Chile: CELADE - División de Población de la CEPAL y Universidad Nacional Autónoma de México.

Jiménez, B., Baillet, L., Ávalos, F., \& Campos, L. (2016). Dependencia funcional y percepción de apoyo familiar en el adulto mayor. Atención Familiar, 23(4), 129-133.

Jiménez, A., Jara, M., \& Miranda, E. (2012). Burnout, apoyo social y satisfacción laboral en docentes. Revista de Psicologia Escolar e Educacional de la Associação Brasileira de Psicologia Escolar e Educacional, 16(1), 125-134. 
Merodio, C., Rivas, V., \& Martínez, A. (2015). Percepción del apoyo familiar y dificultades relacionadas con la diabetes en el adulto mayor. Horizonte Sanitario, 14(1), 51-62.

Ministerio de Salud de la República de Colombia, Minsalud. (1993). Resolución 8430 de 1993: Por la cual se establecen las normas científicas, técnicas y administrativas para la investigación en salud. Bogotá: Ministerio de Salud de la República de Colombia.

Ministerio de Salud de la República de Colombia, Minsalud. (2015). SABE Colombia 2015: Estudio nacional de salud, bienestar y envejecimiento. Bogotá: Ministerio de Salud y Protección Social.

Organización Mundial de la Salud, oms. (2018). Envejecimiento y salud. Ginebra, Suiza. Disponible en: https://www.who.int/es/newsroom/fact-sheets/detail/envejecimiento-ysalud: Organización Mundial de la Salud.
Pereira, T. \& Pavarini, S. (2012). Relación entre apoyo social y capacidad funcional de los mayores con los cambios cognitivos. Enfermagem, 20(4), 1-8.

Polizzi, L. \& Arias, C. (2014). Los vínculos que brindan mayor satisfacción en la red de apoyo social de los adultos mayores. Pensando Psicología, 10(17), 61-70.

Vivaldi, F. \& Barra, E. (2012). Bienestar Psicológico, Apoyo Social Percibido y Percepción de Salud en Adultos Mayores. Terapia Psicológica, 30(2), 23-29.

Zimet G, D., Dahlem, N. W., Zimet, S. G., \& Farley, G. K. (1988). The Multidimensional Scale of Perceived Social Support. Journal of Personality Assessment, 52(1), 30-41. 